\title{
Quantifying Strain and Dislocation Density at Nanocube Interfaces after Assembly and Epitaxy
}

Harshal Agrawal, ${ }^{\S}$ Biplab K. Patra, ${ }^{\S}$ Thomas Altantzis, Annick De Backer, and Erik C. Garnett*

Cite This: ACS Appl. Mater. Interfaces 2020, 12, 8788-8794

Read Online

ABSTRACT: Nanoparticle self-assembly and epitaxy are utilized extensively to make $1 \mathrm{D}$ and 2D structures with complex shapes. High-resolution transmission electron microscopy (HRTEM) has shown that single-crystalline interfaces can form, but little is known about the strain and dislocations at these interfaces. Such information is critically important for applications: drastically reducing dislocation density was the key breakthrough enabling widespread implementation of light-emitting diodes, while strain engineering has been fundamental to modern high-performance transistors, solar cells, and thermoelectrics. In this work, the interfacial defect and strain formation after selfassembly and room temperature epitaxy of $7 \mathrm{~nm}$ Pd nanocubes capped with polyvinylpyrrolidone (PVP) is examined. It is observed that, during ligand removal, the cubes move over large distances

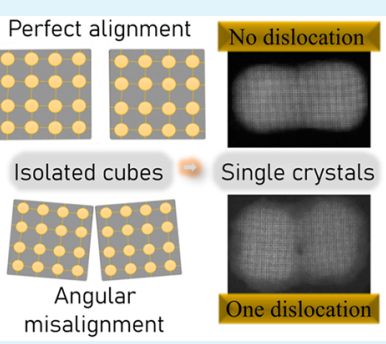
on the substrate, leading to both spontaneous self-assembly and epitaxy to form single crystals.

Subsequently, atomically resolved images are used to quantify the strain and dislocation density at the epitaxial interfaces between cubes with different lateral and angular misorientations. It is shown that dislocation- and strain-free interfaces form when the nanocubes align parallel to each other. Angular misalignment between adjacent cubes does not necessarily lead to grain boundaries but does cause dislocations, with higher densities associated with larger rotations.

KEYWORDS: self-assembly, epitaxy, interface, single crystals, strain, dislocations, high-resolution imaging, nanocubes

\section{INTRODUCTION}

Self-assembly of nanocrystals and successive epitaxy by either dissolving the capping ligands in a suitable solvent or via a chemical trigger has emerged as a promising route to make single crystals with complex nanoscale geometries. ${ }^{1-10}$ This approach is very flexible, including variants where particles are both assembled and epitaxially connected in solution-usually called oriented attachment ${ }^{7,9,11-15}$ —or where assembly first occurs at an interface (liquid-liquid, liquid-solid, or liquidvapor) ${ }^{1,9,10,16-25}$ followed by the epitaxy step (thermal, chemical, or optical). ${ }^{3,4,10,15,26-30}$ Interfacial assembly via aforementioned techniques can lead to $2 \mathrm{D}$ films with nanoscale patterns determined by the shape and surface energetics of the particle, such as a $2 \mathrm{D}$ honeycomb lattice, or to more complex nanopatterns formed by capillary assembly. ${ }^{1,21,31-33}$ In all cases, the choice of materials is broad with a variety of metals, semiconductors, and dielectrics

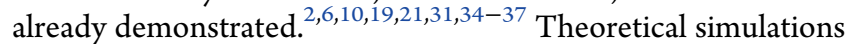
and in situ observation of assembly using high-resolution transmission electron microscopy (HRTEM) and X-ray scattering studies have helped provide insights into the mechanism of assembly and epitaxial connection. ${ }^{32,38-44}$

Despite this vast amount of work in the field of oriented attachment and growth, little is known about the interfacial strain and dislocations at the interfaces after epitaxy. ${ }^{45-48}$ One study showed that $\mathrm{PbSe}$ truncated octahedral nanocrystals with large angular misalignment can have up to seven edge dislocations after initial attachment that can, so far, only be removed with annealing at elevated $\left(\sim 100{ }^{\circ} \mathrm{C}\right)$ temperatures. ${ }^{39}$ Epitaxial particles with perfect angular alignment might be free from strain and dislocations, but this is yet to be probed experimentally. Even with perfect angular alignment, strainand defect-free epitaxy would require either perfect atomic alignment (no lateral displacement) during assembly or some particle mobility during epitaxy to provide atomic registry. Understanding the formation of strain and dislocations at these interfaces is critically important to assess suitability for applications. For example, removing dislocations from epitaxial GaN films was the key breakthrough in enabling widespread implementation of light-emitting diodes, ${ }^{49}$ while strain engineering is used extensively in modern transistors to improve the mobility of the channel. ${ }^{50}$ Strain and dislocations also play a fundamentally important role in other devices, including photovoltaics and thermoelectrics. ${ }^{51-55}$

In this work, we use high-angle annular dark-field scanning transmission electron microscopy (HAADF-STEM) to characterize dislocation and strain formation during nanocube selfassembly and epitaxy at room temperature. Nanocubes, sometimes showing slightly rounded edges or elongation in one direction, are chosen over octahedron, decahedron,

Received: September 30, 2019

Accepted: January 24, 2020

Published: January 24, 2020 
(a) Before epitaxy
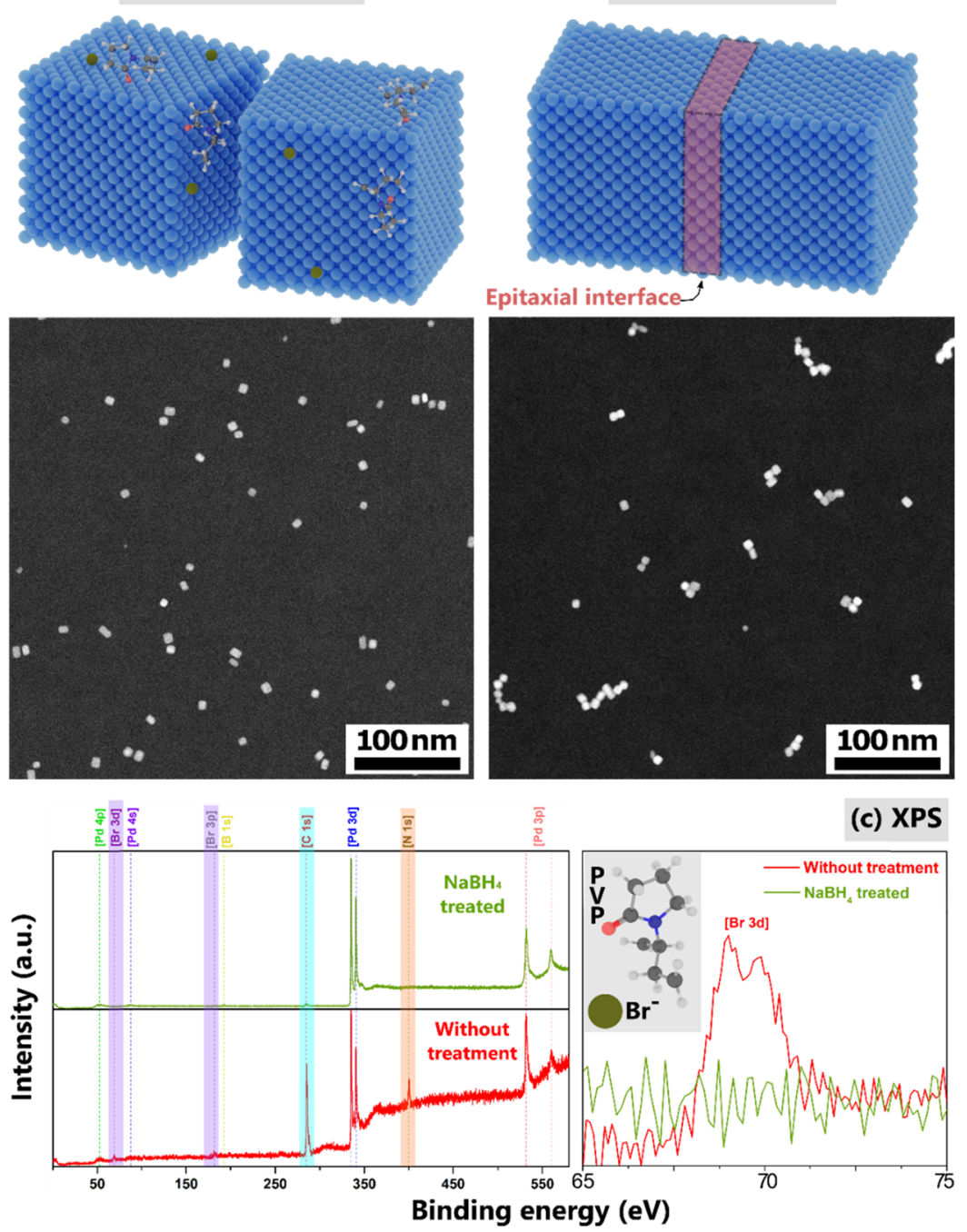

(b) After epitaxy

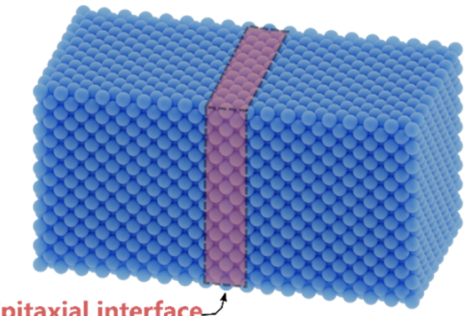

Epitaxial interface-

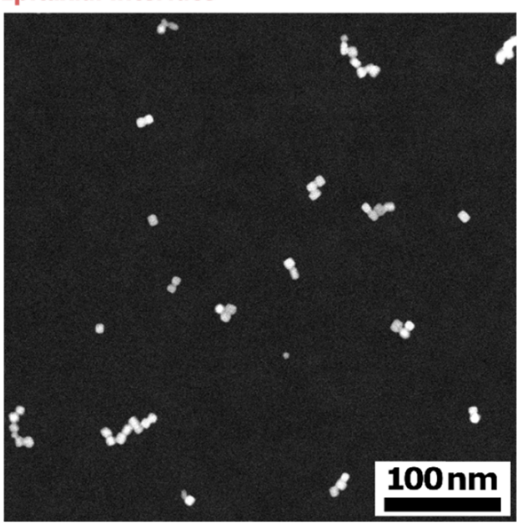

(c) XPS HAADF-STEM image before epitaxy. The surface of the cube is populated with $\mathrm{Br}^{-}$(green) and PVP, which binds either via nitrogen (blue) or carbonyl oxygen atom (red). In the schematic, only a few molecules of capping ligands (PVP monomer and Br ${ }^{-}$) are shown on the surface of cubes for the simplicity of the illustration. (b) Schematic and the corresponding HAADF-STEM image of cubes after epitaxy (after treatment with 0.1 M $\mathrm{NaBH}_{4}$ for $15 \mathrm{~min}$ ). (c) XPS spectra showing the surface chemical composition of the cubes without (red) and after (green) $\mathrm{NaBH}$ treatment. The spectrum on the right is the high-resolution scan in the range of 65 to $75 \mathrm{eV}$ of the main spectrum, and the inset shows the schematic of the binding ligands.

icosahedron, and other morphologies as the model system because of their atomically flat and chemically equivalent faces, which simplify both assembly and atomic merging. ${ }^{38,39,48,56}$ The strain and number of dislocations at the interface of epitaxially connected $\sim 7 \mathrm{~nm}$ palladium (Pd) nanocubes capped with PVP are quantified from the HAADF-STEM images using a model-based image quantification approach implemented in the StatSTEM software. ${ }^{57}$ After dropcasting Pd nanocubes on the substrate, we observed that they move up to hundreds of nanometers during the PVP removal with sodium borohydride $\left(\mathrm{NaBH}_{4}\right)$, enabling spontaneous selfassembly. We confirm that this surface mobility allows for the formation of single-crystalline interfaces with strain values below $5 \%$ and no signs of dislocations. In cases where particles have larger angular misalignment, we observed a higher dislocation density and strain.

\section{RESULTS AND DISCUSSION}

Nanoparticle assembly and epitaxy have been utilized in the past to make $1 \mathrm{D}$ and $2 \mathrm{D}$ structures by stitching together small nanocrystals as the building blocks. In this work, when monocrystalline $7 \mathrm{~nm}$ Pd cubes (Figures S1 and S2) are treated with $\mathrm{NaBH}_{4}$, the cubes migrate over substantial distances on the substrate, transforming from isolated particles to assembled clusters. The cubes self-assemble into a variety of geometries like lines, L shapes, triangles, etc., as shown in Figure 1a,b. During the epitaxy step, $\mathrm{NaBH}_{4}$ acts as the hydride donor that displaces the shape controlling ligands $\mathrm{Br}^{-}$and PVP from the surface of the Pd nanocubes. ${ }^{58-61}$ The surfacesensitive X-ray photoelectron spectroscopy (XPS) characterization data in Figure 1c clearly reveal that, after the $\mathrm{NaBH}_{4}$ treatment, the $\mathrm{N}$ (orange) and $\mathrm{Br}$ (purple) peaks are missing. The $\mathrm{O}$ peak overlaps with $\mathrm{Pd} 3 \mathrm{p}$ and, hence, cannot be quantified. The major peaks in the XPS scan after epitaxy (green) are from $\mathrm{Pd}$ metal, with a minor peak from residual C, 
indicating that most of the surface ligands used during synthesis are eliminated. ${ }^{61}$ This surface ligand removal enables the high particle surface mobility and translation along the substrate, but at the same time, may contribute to some particles partially losing their cubic shape (Figure 2 and Figure S3a,b).

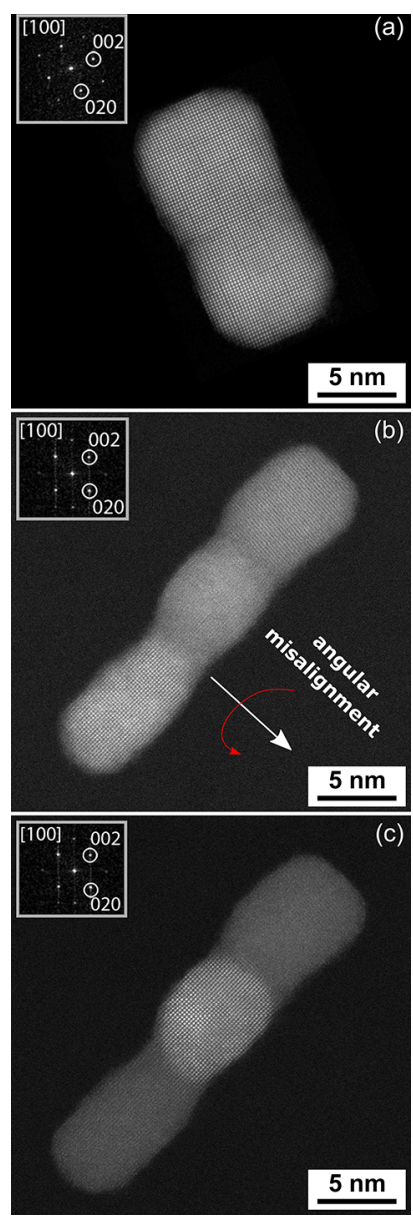

Figure 2. HAADF-STEM images of epitaxial assemblies (a) with perfect angular alignment and $(\mathrm{b}, \mathrm{c})$ with $3.5^{\circ}$ angular misalignment. The micrograph in panel (c) was obtained by rotating the assembly in panel (b) with $3.5^{\circ}$ along the direction indicated by the red arrow, indicating an epitaxial connection between the cubes. The Fourier transforms of the epitaxially connected cubes are shown as insets in all the micrographs.

One striking feature of the ligand-removal process is that, in the same step, the connection between adjacent cubes results in atomic alignment and epitaxy, leading to the formation of a single crystal (Figure 2 and Figure S3). Predominantly, nanocubes join along the $\{100\}$ facets with differing degrees of orientational alignment. Thanks to their cubic shape and the presence of the flat $\{100\}$ facets, imaging of assembled cubes is mainly performed along a $<100>$ zone axis without tilting our sample significantly. In Figure S3a, an image of an assembled couple along a $\langle 110\rangle$ zone axis is also provided. From Figure $2 \mathrm{~b}, \mathrm{c}$, it is prevalent that even a $3.5^{\circ}$ misalignment of adjacent cubes resulted in an epitaxial connection.

Epitaxy between nanocrystals with small angular misorientations has been observed previously to lead to dislocations as would be expected, ${ }^{48}$ but dislocations and strain in epitaxially connected nanocubes without angular misorienta- tion has not been studied. Even for two nanocubes with a perfect angular alignment and the same lattice constant, lateral displacements could lead to dislocations or strain. It is also possible that, during epitaxy, the particles move into perfect atomic alignment to avoid such dislocation or strain formation, leading to the preferential formation of perfect interfaces. To differentiate between these two possibilities, we use nanocubes, which are characterized by six chemically equivalent and atomically flat faces, making them an ideal system for our study where the lower energy of face-to-face alignment increases the chances of perfect angular alignment. The surface atom mobility and cubic shape distortion enabled by ligand removal could reduce the efficacy of the assembly. However, since we still see a high fraction of aligned clusters, this shape distortion likely occurs primarily after particle attachment and during epitaxy.

Local strain and dislocations at the epitaxial nanocube interface were studied using the StatSTEM software. When two cubes come together with perfect angular alignment during epitaxy, they can merge such that there are no dislocations at the interface (Figure 3 and Figures S4 and S5). A small angular misalignment of $2^{\circ}$ can lead to an epitaxial connection with the presence of one dislocation with locally higher strain values (Figure 4). We also verified that a larger angular misalignment of $4^{\circ}$ leads to a higher dislocation density (Figure S6). Thus, in patterns assembled from perfect monocrystalline building blocks, dislocation formation is controlled by the angular misalignment. Besides enabling high surface mobility that is beneficial in reaching the ideal angular alignment, our $\mathrm{NaBH}_{4}$ ligand removal process is also much faster than previous approaches. For example, relying on ligand solubility to trigger the fusing requires up to $16 \mathrm{~h}$, 3,21 while $\mathrm{NaBH}_{4}$ can eliminate the surface capping ligands within $1 \mathrm{~min}$.

Nanocube epitaxy requires two separate steps, and both depend on ligand removal. First, the cubes must overcome the van der Waals attraction with the substrate, allowing for sufficient surface mobility to enable rapid surface diffusion even at room temperature. This allows for the isolated nanocubes formed after dropcasting to come together to form the observed clusters. Such a large-scale movement $(\sim 100 \mathrm{~nm})$ can be explained by partial removal of the surface ligands that bind to the substrate, along with the local evolution of the hydrogen generated when $\mathrm{NaBH}_{4}$ is dissolved in water. $^{62}$ During clustering, nanocubes will attempt to maximize the area of overlap between the $\{100\}$ faces to reduce the surface energy of the system, providing a driving force for angular alignment such that cubes are oriented along the same crystallographic axis. This explains the predominance of short lines and L-shapes observed after $\mathrm{NaBH}_{4}$ treatment. As the nanocube cluster grows, its surface diffusion will also slow down dramatically, which can explain the small number of cubes observed in most clusters.

Once the nanocubes have formed clusters, the atoms at the surface must also rearrange to allow for the epitaxial connection to be observed. Here again, surface ligands play a key role, with as little as $15 \%$ removal dramatically decreasing the interparticle spacing, with the potential minimum occurring at interparticle contact. ${ }^{63}$ At such small distances, epitaxial connections occur to minimize the free energy of the system. $4,5,48,56,63,64$ The potential for eliminating dangling bonds leads to a strong enthalpic driving force; in the case 

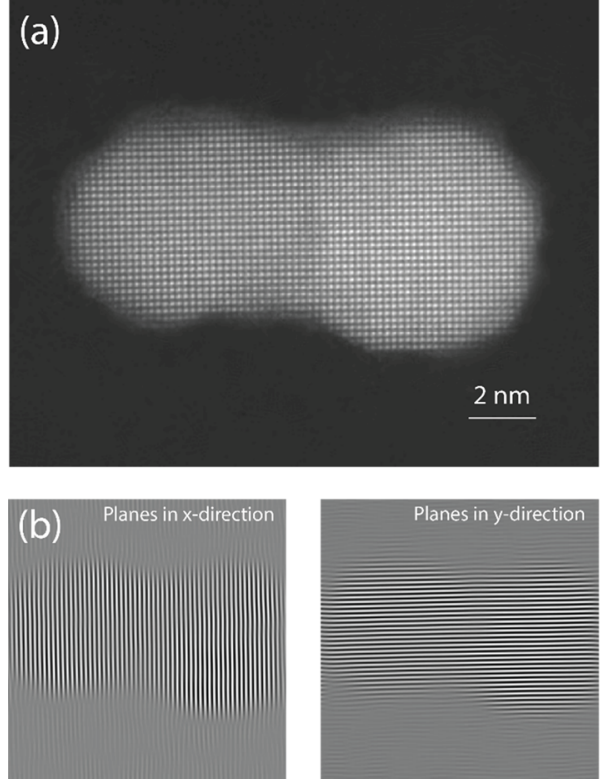

(c)

$\varepsilon_{x x}$

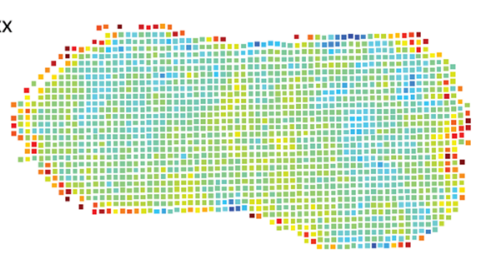

$\varepsilon_{\text {yy }}$
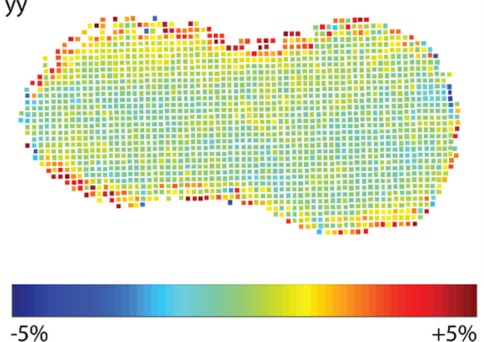

Figure 3. Dislocation and strain analysis of two epitaxial cubes with no dislocation. (a) HAADF-STEM image of the epitaxial cubes. (b) Inverse Fourier transform images showing the individual planes along the $x$ and $y$ directions, illustrating the absence of any dislocation. (c) Strain maps computed for $x\left(\varepsilon_{x x}\right)$ and $y\left(\varepsilon_{y y}\right)$ directions, indicating the absence of strain at the interface of the two cubes.
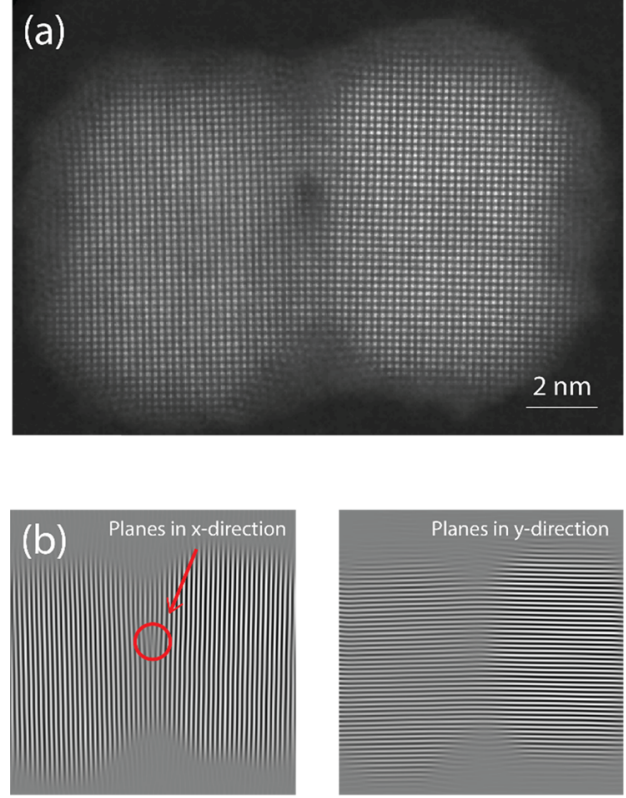

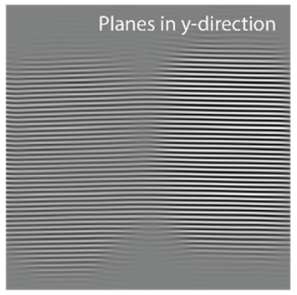

(c)
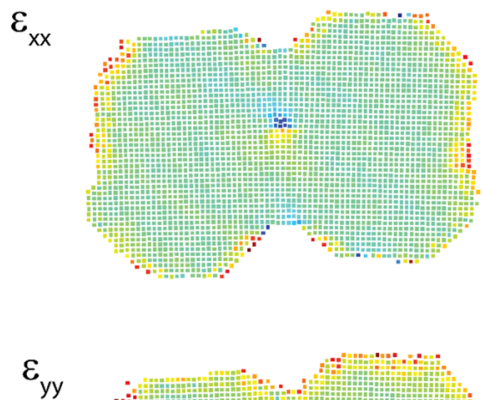

$\varepsilon_{\text {yy }}$

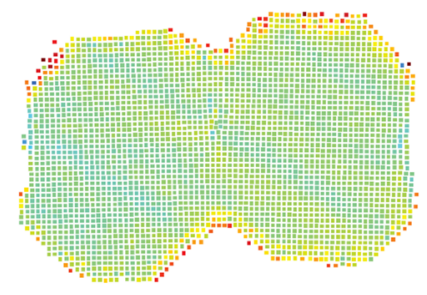

$-20 \%$
$+20 \%$

Figure 4. Dislocation and strain analysis of two epitaxial cubes with a $2^{\circ}$ angular misalignment and one dislocation. (a) HAADF-STEM image of the epitaxial cubes. (b) Inverse Fourier transform images showing the individual planes along the $x$ and $y$ directions, illustrating the presence of one dislocation, indicated by the red arrow. (c) Strain maps computed for $x\left(\varepsilon_{x x}\right)$ and $y\left(\varepsilon_{y y}\right)$ directions.

of $7 \mathrm{~nm}$ Pd cubes, more than 1000 atomic bridges per $\{100\}$ face will be formed.

Stress and dislocation formation in perfect monocrystalline Pd particles depend on the alignment between adjacent cubes after self-assembly. With perfect angular and lateral alignment between cubes, it is verified experimentally that perfect strainand dislocation-free connections can form. The formation of such strain- and dislocation-free interfaces is not expected only with perfect angular alignment. This is because, after assembly, the atoms at opposite sides of the interface should have random lateral displacements, leading to a mismatch of up to half a lattice period, which must be accompanied by strain, dislocations, or both. Therefore, our observation of strain- and dislocation-free epitaxial interfaces requires that nanocubes move laterally, at least over small (sub-nm) distances during the epitaxial connection process itself.

Interestingly, it is observed that the ligand removal can also form a single crystal even if the cubes are not perfectly aligned. A local movement of atoms within misaligned cubes can still lead to an epitaxial connection (Figure 2b,c and Figure S7). The driving force for this local rearrangement of atoms, which also contributes to the change in shape of the cubes, is to avoid the strain caused by the formation of unaligned connections. This atomic surface diffusion process allows for at least a few 
degrees $\left(4^{\circ}\right)$ of angular misalignment (Figure $2 b, c$ and Figure S6) between cubes without leading to a grain boundary formation. However, in the cases where local movement is still not enough to form fully strain free connections as a result of lateral or angular misalignments, the system compensates by nucleating dislocations at the interface to accommodate the strain energy. Figure 4 shows an example of a pair of nanocubes with a $2^{\circ}$ angular misalignment, which leads to a single dislocation. Although the strain is generally close to zero throughout the epitaxial pair (except at the surface), locally around the dislocation high strain values of $10-20 \%$ exist, as expected. Although we do not have enough statistics to make a quantitative correlation between misorientation angle and dislocation density, we do observe that an epitaxial pair with a higher misorientation angle of $4^{\circ}$ has substantially more (five) dislocations (Figure S6). This is very different from the results seen when bending single-crystalline, twinned $\mathrm{Ni}$ nanowires with similar dimensions $(1-6 \mathrm{~nm})$, which were able to accommodate very large lattice rotation angles of up to $38.5^{\circ}$ (shear strain up to $34.6 \%$ ) without nucleating either grain boundaries or dislocations. ${ }^{65}$ We suspect that the drastic difference from our results, where dislocations formed already at rotation angles as little as $2^{\circ}$, arises from the difference between bending a preformed single crystal (twinned in the case of $\mathrm{Ni}$ nanowires) versus epitaxially connecting separate nanocubes with a rotation angle between them. In our case, the two particles that start with relaxed lattices and strain or dislocations will nucleate from the interface during epitaxy, while in the preformed nanowires, the strain builds up continuously over a much larger area and dislocations must nucleate at the nanowire surface. In our system, the nucleation energy for dislocations appears to be lower, which seems to lead to a higher dislocation density but lower strain compared to the preformed nanowires, although further work is needed to quantify these differences. In the case of already perfectly aligned cubes, no rearrangement of atoms is needed to form strain- and dislocation-free epitaxial connections, and as a result, they also retain their near cubic shape (Figure 2a).

\section{CONCLUSIONS}

We have studied the strain and dislocations generated at the interface of assembled and epitaxially connected $7 \mathrm{~nm} \mathrm{Pd}$ cubes. Aqueous $\mathrm{NaBH}_{4}$ solution plays a key role in both the spontaneous nanoparticle assembly and epitaxy steps by promoting the removal of the surface capping ligands at room temperature within a minute. The mobility of both the nanocubes on the substrate surface and $\mathrm{Pd}$ atoms on the nanocube surface enables the formation of dislocation-free single crystals when cubes show perfect angular alignment. The lack of strain and dislocations at this interface indicates that cubes must also be able to move along the substrate during the epitaxial attachment. For an angular misalignment of up to $4^{\circ}$, epitaxial connections between adjacent cubes still form but with higher strain and dislocation density. Given that the crystal quality of assembled and epitaxially connected nanocubes (or particles in oriented attachment) can be comparable to bulk single crystals, such an approach is very promising for applications.

\section{EXPERIMENTAL SECTION}

Preparation of the Precursor. $\mathrm{Na}_{2} \mathrm{PdCl}_{4}(57 \mathrm{mg})$ was dissolved in Milli-Q water $(3 \mathrm{~mL})$. The dissolution takes a long time, so mild heating with periodic ultrasonication followed by vortexing is recommended.

Synthesis. PVP (105 mg), L-ascorbic acid (60 mg), KBr (5 mg), $\mathrm{KCl}(185 \mathrm{mg})$, and Milli-Q water $(8 \mathrm{~mL})$ were placed in a vial $(20$ $\mathrm{mL}$ ) and preheated at $80{ }^{\circ} \mathrm{C}$ for $10 \mathrm{~min}$ in a silicone oil bath with magnetic stirring at $300 \mathrm{rpm}$. The vial was loosely caped from the top. Previously prepared $\mathrm{Na}_{2} \mathrm{PdCl}_{4}(3 \mathrm{~mL})$ was injected in the reaction vessel and left for $3 \mathrm{~h}$; the vial was capped from the top with paraffin. After $3 \mathrm{~h}$, the reaction vessel was removed from the oil bath and left for air convection cooling. After synthesis, the solution was centrifuged at $5000 \mathrm{rpm}$ for $10 \mathrm{~min}$ to settle the coarse particles. The supernatant was centrifuged at 50,000 rpm for $30 \mathrm{~min}$ to isolate nicely faceted $\sim 7 \mathrm{~nm}$ cubic Pd particles and dispersed in ethanol.

Chemical Epitaxy. The sample was prepared by dropcasting the colloidal solution $(10 \mu \mathrm{L})$ on a $\mathrm{Si}_{3} \mathrm{~N}_{4}$ grid. The ligand removal and epitaxy were performed by immersing the grid in $\mathrm{NaBH}_{4}(0.0189 \mathrm{~g}$ in $5 \mathrm{~mL}$ water, $0.1 \mathrm{M})$ solution without any stirring. After $15 \mathrm{~min}$, the grid was cleaned in a solution of $1: 1$ ratio by volume of water and ethanol for $\sim 2 \mathrm{~h}$ with stirring at $150 \mathrm{rpm}$. It should be noted that, each time, a fresh solution of $\mathrm{NaBH}_{4}$ was used for ligand removal and epitaxy.

Dislocation Visualization. Potential dislocations are visualized by showing the individual lattice planes in the $x$ and $y$ directions. To visualize these, first, a Fourier transform is obtained from the HAADF-STEM image. Next, two symmetric lattice points in the $x$ or $y$ direction are masked from this Fourier transform. The inverse Fourier transform of these masked reflections results in a visualization of the potential dislocations, which might appear at the interface.

Strain Maps. The strain maps are obtained using the StatSTEM software. By modeling images as a superposition of Gaussian functions located at the atomic columns, the atomic column positions can be measured with high accuracy and precision. A direct comparison of the measured column positions with the expected column positions of an ideal crystal lattice gives the displacement of the atomic columns. By using the first derivative, these measured displacement vectors can be used to compute atomically resolved strain maps.

\section{ASSOCIATED CONTENT}

\section{Supporting Information}

The Supporting Information is available free of charge at https://pubs.acs.org/doi/10.1021/acsami.9b17779.

Experimental details of the chemical synthesis and characterizations, chemical epitaxy, strain visualization specifics, photographs of nanocube characterization along with XRD, HAADF-STEM images of epitaxially connected pairs of cubes, and strain maps of the epitaxially connected couples of nanocubes (PDF)

\section{AUTHOR INFORMATION}

\section{Corresponding Author}

Erik C. Garnett - Center for Nanophotonics, AMOLF, 1098 XG Amsterdam, The Netherlands; Email: garnett@amolf.nl

\section{Authors}

Harshal Agrawal - Center for Nanophotonics, AMOLF, 1098 XG Amsterdam, The Netherlands; orcid.org/0000-00025106-3947

Biplab K. Patra - Center for Nanophotonics, AMOLF, 1098 XG Amsterdam, The Netherlands; 이이.org/0000-00030592-4344

Thomas Altantzis - Electron Microscopy for Materials Research (EMAT), University of Antwerp, Antwerp 2020, Belgium; (1) orcid.org/0000-0002-4940-7931 
Annick De Backer - Electron Microscopy for Materials

Research (EMAT), University of Antwerp, Antwerp 2020,

Belgium; (1) orcid.org/0000-0002-8592-4776

Complete contact information is available at:

https://pubs.acs.org/10.1021/acsami.9b17779

\section{Author Contributions \\ ${ }^{\S}$ H.A. and B.K.P. contributed equally. H.A. carried out the synthesis and welding experiments along with B.K.P under the supervision of E.C.G. T.A. and A.D.B. did all the high- resolution imaging and strain/dislocation analysis. H.A. wrote the manuscript with contributions from all the authors. All authors discussed the results.}

\section{Notes}

The authors declare no competing financial interest.

\section{ACKNOWLEDGMENTS}

The work at AMOLF is part of the research program of the "Nederlandse Organisatie voor Wetenschappelijk Onderzoek" (NWO). This work was supported by the NWO VIDI grant (project no. 14846). The authors would like to thank Reinout Jaarsma and Dr. Sven Askes for helping with the XPS measurements. A.D.B. acknowledges a postdoctoral grant from the research foundation Flanders (FWO). The authors acknowledge financial support from the European Commission under the Horizon 2020 Programme by means of the grant agreement no. 731019 EUSMI.

\section{REFERENCES}

(1) Evers, W. H.; Goris, B.; Bals, S.; Casavola, M.; de Graaf, J.; van Roij, R.; Dijkstra, M.; Vanmaekelbergh, D. Low-Dimensional Semiconductor Superlattices Formed by Geometric Control over Nanocrystal Attachment. Nano Lett. 2013, 13, 2317-2323.

(2) Tang, Z.; Kotov, N. A.; Giersig, M. Spontaneous Organization of Single CdTe Nanoparticles into Luminescent Nanowires. Science 2002, 297, 237-240.

(3) Baumgardner, W. J.; Whitham, K.; Hanrath, T. Confined-butConnected Quantum Solids via Controlled Ligand Displacement. Nano Lett. 2013, 13, 3225-3231.

(4) Sandeep, C. S. S.; Azpiroz, J. M.; Evers, W. H.; Boehme, S. C.; Moreels, I.; Kinge, S.; Siebbeles, L. D. A.; Infante, I.; Houtepen, A. J. Epitaxially Connected PbSe Quantum-Dot Films: Controlled Neck Formation and Optoelectronic Properties. ACS Nano 2014, 8, 11499-11511.

(5) Whitham, K.; Smilgies, D. M.; Hanrath, T. Entropic, Enthalpic, and Kinetic Aspects of Interfacial Nanocrystal Superlattice Assembly and Attachment. Chem. Mater. 2018, 30, 54-63.

(6) Nakagawa, Y.; Kageyama, H.; Oaki, Y.; Imai, H. Formation of Monocrystalline 1D and 2D Architectures via Epitaxial Attachment: Bottom-Up Routes through Surfactant-Mediated Arrays of Oriented Nanocrystals. Langmuir 2015, 31, 6197-6201.

(7) Cho, K. S.; Talapin, D. V.; Gaschler, W.; Murray, C. B. Designing $\mathrm{PbSe}$ Nanowires and Nanorings through Oriented Attachment of Nanoparticles. J. Am. Chem. Soc. 2005, 127, 71407147.

(8) Schliehe, C.; Juarez, B. H.; Pelletier, M.; Jander, S.; Greshnykh, D.; Nagel, M.; Meyer, A.; Foerster, S.; Kornowski, A.; Klinke, C.; et al. Ultrathin $\mathrm{PbS}$ Sheets by Two-Dimensional Oriented Attachment. Science 2010, 329, 550-553.

(9) Tong, Y.; Bohn, B. J.; Bladt, E.; Wang, K.; Müller-Buschbaum, P.; Bals, S.; Urban, A. S.; Polavarapu, L.; Feldmann, J. From Precursor Powders to CsPbX 3 Perovskite Nanowires: One-Pot Synthesis, Growth Mechanism, and Oriented Self-Assembly. Angew. Chem., Int. Ed. 2017, 56, 13887-13892.
(10) Sciacca, B.; Berkhout, A.; Brenny, B. J. M.; Oener, S. Z.; van Huis, M. A.; Polman, A.; Garnett, E. C. Monocrystalline Nanopatterns Made by Nanocube Assembly and Epitaxy. Adv. Mater. 2017, 29, 1701064.

(11) Sarkar, S.; Acharya, S.; Chakraborty, A.; Pradhan, N. Zinc Blende 0D Quantum Dots to Wurtzite 1D Quantum Wires: The Oriented Attachment and Phase Change in ZnSe Nanostructures. J. Phys. Chem. Lett. 2013, 4, 3292-3297.

(12) Kao, L. C.; Ye, Y.; Liu, Y.-S.; Dong, C. L.; Guo, J.; Liou, S. Y. H. A Facile Route for the Synthesis of Heterogeneous Crystal Structures in Hierarchical Architectures with Vacancy-Driven Defects via the Oriented Attachment Growth Mechanism. J. Mater. Chem. A 2018, 6, 10663-10673.

(13) Yu, J. H.; Joo, J.; Park, H. M.; Baik, S.-I.; Kim, Y. W.; Kim, S. C.; Hyeon, T. Synthesis of Quantum-Sized Cubic ZnS Nanorods by the Oriented Attachment Mechanism. J. Am. Chem. Soc. 2005, 127, 5662-5670.

(14) Yuwono, V. M.; Burrows, N. D.; Soltis, J. A.; Penn, R. L. Oriented Aggregation: Formation and Transformation of Mesocrystal Intermediates Revealed. J. Am. Chem. Soc. 2010, 132, 2163-2165.

(15) Figuerola, A.; Franchini, I. R.; Fiore, A.; Mastria, R.; Falqui, A.; Bertoni, G.; Bals, S.; van Tendeloo, C.; Kudera, S.; Cingolani, R.; et al. End-to-End Assembly of Shape-Controlled Nanocrystals via a Nanowelding Approach Mediated by Gold Domains. Adv. Mater. 2009, 21, 550-554.

(16) Hanske, C.; Hill, E. H.; Vila-Liarte, D.; González-Rubio, G.; Matricardi, C.; Mihi, A.; Liz-Marzán, L. M. Solvent-Assisted SelfAssembly of Gold Nanorods into Hierarchically Organized Plasmonic Mesostructures. ACS Appl. Mater. Interfaces 2019, 11, 11763-11771.

(17) Kister, T.; Maurer, J. H. M.; González-García, L.; Kraus, T. Ligand-Dependent Nanoparticle Assembly and Its Impact on the Printing of Transparent Electrodes. ACS Appl. Mater. Interfaces 2018, 10, 6079-6083.

(18) Xin, B.; Pak, Y.; Mitra, S.; Almalawi, D.; Alwadai, N.; Zhang, Y.; Roqan, I. S. Self-Patterned CsPbBr 3 Nanocrystals for HighPerformance Optoelectronics. ACS Appl. Mater. Interfaces 2019, 11, 5223-5231.

(19) Boneschanscher, M. P.; Evers, W. H.; Geuchies, J. J.; Altantzis, T.; Goris, B.; Rabouw, F. T.; Van Rossum, S. A. P.; Van Der Zant, H. S. J.; Siebbeles, L. D. A.; Van Tendeloo, G.; et al. Long-Range Orientation and Atomic Attachment of Nanocrystals in 2D Honeycomb Superlattices. Science 2014, 344, 1377-1380.

(20) Van Overbeek, C.; Peters, J. L.; Van Rossum, S. A. P.; Smits, M.; Van Huis, M. A.; Vanmaekelbergh, D. Interfacial Self-Assembly and Oriented Attachment in the Family of $\mathrm{PbX}(\mathrm{X}=\mathrm{S}, \mathrm{Se}, \mathrm{Te})$ Nanocrystals. J. Phys. Chem. C 2018, 122, 12464-12473.

(21) Peters, J. L.; Altantzis, T.; Lobato, I.; Jazi, M. A.; Van Overbeek, C.; Bals, S.; Vanmaekelbergh, D.; Sinai, S. B. Mono- and Multilayer Silicene-Type Honeycomb Lattices by Oriented Attachment of PbSe Nanocrystals: Synthesis, Structural Characterization, and Analysis of the Disorder. Chem. Mater. 2018, 30, 4831-4837.

(22) Kovalenko, M. V.; Protesescu, L.; Bodnarchuk, M. I. Properties and Potential Optoelectronic Applications of Lead Halide Perovskite Nanocrystals. Science 2017, 358, 745-750.

(23) Tao, A.; Sinsermsuksakul, P.; Yang, P. Tunable Plasmonic Lattices of Silver Nanocrystals. Nat. Nanotechnol. 2007, 2, 435-440.

(24) Kuemin, C.; Nowack, L.; Bozano, L.; Spencer, N. D.; Wolf, H. Oriented Assembly of Gold Nanorods on the Single-Particle Level. Adv. Funct. Mater. 2012, 22, 702-708.

(25) Tao, A. R.; Huang, J.; Yang, P. Langmuir - Blodgettry of Nanocrystals and Nanowires. Acc. Chem. Res. 2008, 41, 1662-1673.

(26) Lee, J.; Lee, I.; Kim, T.-S.; Lee, J.-Y. Efficient Welding of Silver Nanowire Networks without Post-Processing. Small 2013, 9, 28872894.

(27) Kim, J.-H.; Kim, S.-R.; Kil, H.-J.; Kim, Y.-C.; Park, J.-W. Highly Conformable, Transparent Electrodes for Epidermal Electronics. Nano Lett. 2018, 18, 4531-4540. 
(28) Lee, J.-Y.; Connor, S. T.; Cui, Y.; Peumans, P. SolutionProcessed Metal Nanowire Mesh Transparent Electrodes. Nano Lett. 2008, 8, 689-692.

(29) Zhong, Z.; Lee, H.; Kang, D.; Kwon, S.; Choi, Y.-M.; Kim, I.; Kim, K.-Y.; Lee, Y.; Woo, K.; Moon, J. Continuous Patterning of Copper Nanowire-Based Transparent Conducting Electrodes for Use in Flexible Electronic Applications. ACS Nano 2016, 10, 7847-7854.

(30) Miszta, K.; De Graaf, J.; Bertoni, G.; Dorfs, D.; Brescia, R.; Marras, S.; Ceseracciu, L.; Cingolani, R.; Van Roij, R.; Dijkstra, M.; et al. Hierarchical Self-Assembly of Suspended Branched Colloidal Nanocrystals into Superlattice Structures. Nat. Mater. 2011, 10, 872876.

(31) Vogt, P.; De Padova, P.; Quaresima, C.; Avila, J.; Frantzeskakis, E.; Asensio, M. C.; Resta, A.; Ealet, B.; Le Lay, G. Silicene: Compelling Experimental Evidence for Graphenelike Two-Dimensional Silicon. Phys. Rev. Lett. 2012, 108, 155501.

(32) Geuchies, J. J.; van Overbeek, C.; Evers, W. H.; Goris, B.; de Backer, A.; Gantapara, A. P.; Rabouw, F. T.; Hilhorst, J.; Peters, J. L.; Konovalov, O.; et al. In Situ Study of the Formation Mechanism of Two-Dimensional Superlattices from PbSe Nanocrystals. Nat. Mater. 2016, 15, 1248-1254.

(33) Kraus, T.; Malaquin, L.; Schmid, H.; Riess, W.; Spencer, N. D.; Wolf, H. Nanoparticle Printing with Single-Particle Resolution. Nat. Nanotechnol. 2007, 2, 570-576.

(34) Flauraud, V.; Mastrangeli, M.; Bernasconi, G. D.; Butet, J.; Alexander, D. T. L.; Shahrabi, E.; Martin, O. J. F.; Brugger, J. Nanoscale Topographical Control of Capillary Assembly of Nanoparticles. Nat. Nanotechnol. 2017, 12, 73-80.

(35) Takasaki, M.; Oaki, Y.; Imai, H. Switchable Oriented Attachment and Detachment of Calcite Nanocrystals. CrystEngComm 2016, 18, 8999-9002.

(36) Wang, L.; Liu, B.; Zhao, X.; Demir, H. V.; Gu, H.; Sun, H. Solvent-Assisted Surface Engineering for High-Performance AllInorganic Perovskite Nanocrystal Light-Emitting Diodes. ACS Appl. Mater. Interfaces 2018, 10, 19828-19835.

(37) Gomez, L.; Lin, J.; de Weerd, C.; Poirier, L.; Boehme, S. C.; von Hauff, E.; Fujiwara, Y.; Suenaga, K.; Gregorkiewicz, T. Extraordinary Interfacial Stitching between Single All-Inorganic Perovskite Nanocrystals. ACS Appl. Mater. Interfaces 2018, 10, 5984-5991.

(38) Li, D.; Nielsen, M. H.; Lee, J. R. I.; Frandsen, C.; Banfield, J. F.; De Yoreo, J. J. Direction-Specific Interactions Control Crystal Growth by Oriented Attachment. Science 2012, 336, 1014-1018.

(39) van Huis, M. A.; Kunneman, L. T.; Overgaag, K.; Xu, Q.j Pandraud, G.; Zandbergen, H. W.; Vanmaekelbergh, D. LowTemperature Nanocrystal Unification through Rotations and Relaxations Probed by in Situ Transmission Electron Microscopy. Nano Lett. 2008, 8, 3959-3963.

(40) Zhu, C.; Liang, S.; Song, E.; Zhou, Y.; Wang, W.; Shan, F.; Shi, Y.; Hao, C.; Yin, K.; Zhang, T.; et al. In-Situ Liquid Cell Transmission Electron Microscopy Investigation on Oriented Attachment of Gold Nanoparticles. Nat. Commun. 2018, 9, 421.

(41) Tan, S. F.; Raj, S.; Bisht, G.; Annadata, H. V.; Nijhuis, C. A.; Král, P.; Mirsaidov, U. Nanoparticle Interactions Guided by ShapeDependent Hydrophobic Forces. Adv. Mater. 2018, 30, 1707077.

(42) Lv, W.; He, W.; Wang, X.; Niu, Y.; Cao, H.; Dickerson, J. H.; Wang, Z. Understanding the Oriented-Attachment Growth of Nanocrystals from an Energy Point of View: A Review. Nanoscale 2014, 6, 2531-2547.

(43) Lv, W.; Zhu, Y.; Niu, Y.; Huo, W.; Li, K.; Zhu, G.; Liang, Y.; $\mathrm{Wu}, \mathrm{W}$; $\mathrm{He}, \mathrm{W}$. Assembly of Anisotropic One Dimensional Ag Nanostructures through Orientated Attachment: On-Axis or off-Axis Growth? RSC Adv. 2015, 5, 20783-20787.

(44) Lv, W.; Huo, W.; Niu, Y.; Zhu, Y.; Xie, Y.; Guo, X.; He, W. Oriented-Attachment Dimensionality Build-up via van Der Waals Interaction. CrystEngComm 2015, 17, 729-733.

(45) Hofmann, F.; Abbey, B.; Liu, W.; Xu, R.; Usher, B. F.; Balaur, E.; Liu, Y. X-Ray Micro-Beam Characterization of Lattice Rotations and Distortions Due to an Individual Dislocation. Nat. Commun. 2013, 4, 2774.

(46) Ondry, J. C.; Hauwiller, M. R.; Alivisatos, A. P. Dynamics and Removal Pathway of Edge Dislocations in Imperfectly Attached $\mathrm{PbTe}$ Nanocrystal Pairs: Toward Design Rules for Oriented Attachment. ACS Nano 2018, 12, 3178-3189.

(47) Tsai, M. H.; Chen, S. Y.; Shen, P. Imperfect Oriented Attachment: Accretion and Defect Generation of Nanosize Rutile Condensates. Nano Lett. 2004, 4, 1197-1201.

(48) Penn, R. L.; Banfield, J. F. Imperfect Oriented Attachment: Dislocation Generation in Defect-Free Nanocrystals. Science 1998, 281, 969-971.

(49) Nakamura, S. The Roles of Structural Imperfections in InGaNBased Blue Light-Emitting Diodes and Laser Diodes. Science 1998, 281, 956-961.

(50) Lee, M. L.; Fitzgerald, E. A.; Bulsara, M. T.; Currie, M. T.; Lochtefeld, A. Strained Si, SiGe, and Ge Channels for High-Mobility Metal-Oxide-Semiconductor Field-Effect Transistors. J. Appl. Phys. 2005, 97, No. 011101.

(51) Hartman, K.; Bertoni, M.; Serdy, J.; Buonassisi, T. Dislocation Density Reduction in Multicrystalline Silicon Solar Cell Material by High Temperature Annealing. Appl. Phys. Lett. 2008, 93, 122108.

(52) Watling, J. R.; Paul, D. J. A Study of the Impact of Dislocations on the Thermoelectric Properties of Quantum Wells in the Si/SiGe Materials System. J. Appl. Phys. 2011, 110, 114508.

(53) Fitzgerald, E. A.; Xie, Y.-H.; Green, M. L.; Brasen, D.; Kortan, A. R.; Michel, J.; Mii, Y.-J.; Weir, B. E. Totally Relaxed $\mathrm{Ge}_{\mathrm{x}} \mathrm{Si}_{1-\mathrm{x}}$ Layers with Low Threading Dislocation Densities Grown on Si Substrates. Appl. Phys. Lett. 1991, 59, 811-813.

(54) Yang, L.; Bulsara, M. T.; Lee, K. E.; Fitzgerald, E. A. Compositionally-Graded InGaAs-InGaP Alloys and GaAsSb Alloys for Metamorphic InP on GaAs. J. Cryst. Growth 2011, 324, 103-109.

(55) Tsao, J. Y.; Han, J.; Haitz, R. H.; Pattison, P. M. The Blue LED Nobel Prize: Historical Context, Current Scientific Understanding, Human Benefit. Ann. Phys. 2015, 527, A53-A61.

(56) Boles, M. A.; Talapin, D. V. Connecting the Dots. Science 2014, 344, 1340-1341.

(57) De Backer, A.; van den Bos, K. H. W.; Van den Broek, W.; Sijbers, J.; Van Aert, S. StatSTEM: An Efficient Approach for Accurate and Precise Model-Based Quantification of Atomic Resolution Electron Microscopy Images. Ultramicroscopy 2016, 171, 104-116.

(58) Koczkur, K. M.; Mourdikoudis, S.; Polavarapu, L.; Skrabalak, S. E. Polyvinylpyrrolidone (PVP) in Nanoparticle Synthesis. Dalton Trans. 2015, 44, 17883-17905.

(59) Chen, T.; Zhang, Y.; Xu, W. Size-Dependent Catalytic Kinetics and Dynamics of Pd Nanocubes: A Single-Particle Study. Phys. Chem. Chem. Phys. 2016, 18, 22494-22502.

(60) Ansar, S. M.; Ameer, F. S.; Hu, W.; Zou, S.; Pittman, C. U., Jr.; Zhang, D. Removal of Molecular Adsorbates on Gold Nanoparticles Using Sodium Borohydride in Water. Nano Lett. 2013, 13, 12261229.

(61) Nalajala, N.; Gooty Saleha, W. F.; Ladewig, B. P.; Neergat, M. Sodium Borohydride Treatment: A Simple and Effective Process for the Removal of Stabilizer and Capping Agents from Shape-Controlled Palladium Nanoparticles. Chem. Commun. 2014, 50, 9365-9368.

(62) Ma, J.; Choudhury, N. A.; Sahai, Y. A Comprehensive Review of Direct Borohydride Fuel Cells. Renewable Sustainable Energy Rev. 2010, 14, 183-199.

(63) Schapotschnikow, P.; Pool, R.; Vlugt, T. J. H. Molecular Simulations of Interacting Nanocrystals. Nano Lett. 2008, 8, 29302934.

(64) Schapotschnikow, P.; van Huis, M. A.; Zandbergen, H. W.; Vanmaekelbergh, D.; Vlugt, T. J. H. Morphological Transformations and Fusion of PbSe Nanocrystals Studied Using Atomistic Simulations. Nano Lett. 2010, 10, 3966-3971.

(65) Wang, L.; Liu, P.; Guan, P.; Yang, M.; Sun, J.; Cheng, Y.; Hirata, A.; Zhang, Z.; Ma, E.; Chen, M.; et al. In Situ Atomic-Scale Observation of Continuous and Reversible Lattice Deformation beyond the Elastic Limit. Nat. Commun. 2013, 4, 2413. 